ISSN 2219-6722 / ISSNE 2222-2707

\title{
UNA MIRADA A LA COMPETITIVIDAD GLOBAL: PRODUCCIÓN DE LA INDUSTRIA ELECTRÓNICA
}

\author{
César H. Ortega Jiménez \\ Universidad Nacional Autónoma de Honduras (UNAH) \\ Facultad de Ciencias Económicas, Administrativas y Contables (FCEAC) \\ Instituto de Investigaciones Económicas y Sociales (IIES) \\ Ciudad universitaria, edificio C2, primer piso, Tel/fax: + (504) 2216-6100 Ext. 100894 \\ ORCID: 0000-0003-1911-9725 \\ cortega@unah.edu.hn \\ *(1) Pedro Garrido Vega \\ Universidad de Sevilla, \\ pgarrido@us.es \\ José Antonio Domínguez Machuca \\ Universidad de Sevilla \\ pgarrido@us.es
}

\section{RESUMEN}

Se estudian dos de los principales programas de producción: Estrategia de producción (por sus siglas en inglés PS) y gestión de tecnología (por sus siglas en inglés TM) de las siguientes dos formas: 1) Testeando y validando los efectos de ajuste de la vinculación entre prácticas de dichos programas; y (2) Explorando el papel que juega el entorno de las fábricas como un inhibidor de ajuste entre PS y TM. El artículo se fundamenta en el análisis estadístico de encuestas, que involucra 88 fábricas de la industria de electrónica, distribuidas entre Alemania, Austria, Canadá, Corea del Sur, España, Estados Unidos, Finlandia, Italia, Japón y Suecia. Con ello, el estudio prueba la existencia del ajuste entre las prácticas de PS y las de TM. Para ello, se proveen argumentaciones analíticas y empíricas, mostrando que la PS y la TM refuerzan mutuamente sus prácticas para lograr una mayor competitividad global, medida por el rendimiento de operaciones. Esto parece indicar que la implementación común de las prácticas de producción estudiadas a través de la industria de la electrónica es importante en los entornos en los cuales operan las fábricas.

El artículo sugiere un patrón de mejora donde la PS y la TM tienen que ser implementados mano a mano para lograr una mejor ventaja de su eficacia. Las articulaciones de prácticas de producción proveen una base sobre el cual el ajuste se origina, extendiendo sus beneficios a través de la organización. La investigación representa un intento de operacionalizar y validar empíricamente las articulaciones entre prácticas de producción en un entorno industrial internacional. Además, el artículo provee recomendaciones originales para los profesionales y decisores en las fábricas, de cómo lograr lo mejor de la implementación de la PS y la TM en la industria de la electrónica internacional.

Palabras clave: Competitividad, programas de producción, electrónica, interrelaciones. 


\title{
A GLANCE AT GLOBAL COMPETITIVENESS: PRODUCTION IN ELECTRONICS INDUSTRY
}

\author{
César H. Ortega Jiménez, \\ Universidad Nacional Autónoma de Honduras (UNAH), \\ Facultad de Ciencias Económicas, Administrativas y Contables (FCEAC), \\ Instituto de Investigaciones Económicas y Sociales (IIES), \\ Ciudad universitaria, edificio C2, primer piso, Tel/fax: + (504) 2216-6100 Ext. 100894 \\ ORCID: 0000-0003-1911-9725 \\ cortega@unah.edu.hn \\ *(1) Pedro Garrido Vega \\ Universidad de Sevilla, \\ pgarrido@us.es \\ José Antonio Domínguez Machuca \\ Universidad de Sevilla, \\ pgarrido@us.es
}

\begin{abstract}
Two of the main production programs (PPs) in manufacturing: Production strategy (PS) and technology management (TM) are here studied twofold: 1) testing and validating fit effects of the links between practices from such programs; and 2) Exploring the role played by the environment of plants as an inhibitor of the PS-TM fit. The paper is based on statistical analysis on a survey that involves 88 plants from the electronics industry, distributed among Austria, Canada, Finland, Germany, Italy, Japan, South Korea, Spain, Sweden, and USA. Hence, the study proves the existence of fit between PS and TM practices. To do so, the paper provides analytical and empirical argumentations showing that PS and TM mutually reinforce each other's practices in order to gain greater competitiveness, measured by operational performance. This may indicate that common implementation of studied production practices across the electronics industry is important in environments in which plants operate.
\end{abstract}

The paper suggests a pattern of improvements where PS and TM have to be implemented hand-in-hand to take better advantage of their effectiveness. Production practices linkages provide ground over which fit originates, spreading its benefits throughout the organization.

The research represents an attempt to operationalize and empirically validate linkages between production practices in an international industrial environment. Furthermore, the paper also provides original suggestions to practitioners and decision maker in plants on how to make the most out of implementing PS and TM in the international electronics industry.

Keywords: Competitiveness, production programs, electronics, interrelations.

1 Corresponding author

6 Revista Economía y Administración (E\&A) / V0L. 8 


\section{INTRODUCCIÓN}

En estudios previos sobre los programas de producción (PP) no evidencia estadística de forma concluyente, sobre cómo la implementación de algún PP de paso a mejoras competitivas en distintos tipos de organizaciones. Por lo que, antes de seleccionar, adaptar -según sea necesario, implementar y vincular un PP adecuado, es necesario también poner en marcha un plan estratégico bien concebido sobre la base de circunstancias de la organización -medio ambiente-. Si lo anterior no se hace, los PP no tendrán el efecto deseado sobre la consecución de la competitividad requerida en los mercados globales. Todo lo anterior debería estar vinculado a una ruta planificada de mejora continua. Estos tres elementos -de contingencia, vínculos y la mejora continua- son en términos generales, el enfoque de la Manufactura de Alto Rendimiento, por sus siglas en inglés (HPM), (Ortega y Eguía, 2010).

En esta búsqueda de la competitividad y la mejora continua, el uso eficaz de los recursos tecnológicos debe ser esencial para el logro de una ventaja competitiva sostenible, que persiga el aumento en el rendimiento de las organizaciones. Por otro lado, aunque se conoce que la aplicación del programa de gestión tecnológica -conjunto de prácticas de tecnología puede en principio aumentar la ventaja competitiva; es necesario analizarlo en combinación con el programa de estrategia de producción -operaciones-, en el contexto de las organizaciones, ya que parece existir una clara influencia entre ellos (Garrido et al., 2015; Ortega et al., 2011; Schroeder y Flynn, 2001). Además, el presente estudio subraya la necesidad de investigar el impacto combinado de ambos programas para distinguir entre dos tipos de fábricas: las de clase mundial -alto rendimiento- y el resto -rendimiento estándar-.

Por lo tanto, para probar la vinculación entre estos dos programas i.-Tecnología y ii.- Estrategia de producción, esta investigación se centra en la industria electrónica como una etapa previa para subsecuentes estudios de sectores industriales y de interrelaciones entre diferentes programas de producción. En este caso, la industria electrónica se caracteriza por la innovación tecnológica, los cambios rápidos y la competencia agresiva en un entorno de mercado global con redes de suministro complejas. En este ambiente, las fuentes de ventaja competitivas pueden venir de las tecnologías y la consolidación de habilidades de la producción que apoyan la generación de productos que la competencia no puede anticipar.

Por otra parte, este sector tiene una de las industrias más dinámicas, influyentes e importantes del mundo, en términos de producción, comercialización, intercambios y creación de riqueza ${ }^{1}$. A pesar de los retos macroeconómicos sustanciales, el sector muestra claros signos de competitividad. Por un lado, la producción de tabletas, laptop/notebook y teléfonos inteligentes (smartphones) muestran los siguientes datos: 1) Las ventas de unidades de la tabletas llegarán a casi 321 millones en 2015 -más del $25 \%$ en relación al 2014, cuando un poco más de 256 millones se vendieron-; 2) Para los laptops -computador portátil- las ventas como unidades seguirán aumentando de un poco más de 308 millones en el 2014, a casi 317 millones en el 2015; y 3) Las ventas de unidades de smartphones llegarán a más de 1.95 billones de unidades en el 2015 de los casi 1.87 billones de unidades en el 2014.

Por otro lado, las ventas de semiconductores de todo el mundo para el 2014 fueron de casi \$ 336 dólares estadounidenses (siendo este el ingreso total en ventas anuales más alto de la industria de todos los tiempos) $)^{2}$. Finalmente, se destaca que la conectividad de la información - "Internet of Things", por sus

\footnotetext{
$1 \mathrm{http}: / /$ techcrunch.com/2014/07/06/gartner-device-shipments-break-2-4bunits-in-2014-tablets-to-overtake-pc-sales-in-2015/ (último acceso 9-10-15) 2 http://www.semiconductors.org/
} 
siglas en inglés (IoT), tendrá un incremento del $29 \%$ en la industria de semiconductores durante los próximos cinco años. La IoT: el próximo factor de crecimiento para la industria de semiconductores, proyecta que la facturación en la industria de semiconductores mundial aumentará a \$ 432 billones de dólares estadounidenses en 2019, correspondiente a una tasa de crecimiento anual compuesto del $5,2 \%$. Dispositivos que puederequerir estar conectados, desde tecnologías orientadas al consumidor como Smartphone, laptops, tabletas, autos y accesorios personales, hasta las grandes maquinarias industriales y comerciales, dependen de los componentes para sensores de recolección de datos; por lo que, la necesidad de estos sensores será uno los mayores conductores de crecimiento en la industria de semiconductores ${ }^{3}$.

Por lo tanto, teniendo en cuenta el contexto global y dinámico del sector de la electrónica, el presente estudio tiene como objetivo posteriormente las interrelaciones eficaces entre los niveles de implementación de ambos programas, estrategia de producción y gestión tecnológica (Aoki et al.,201; Phan et al. 2011). Así, la pregunta de investigación que surge aquí y sirve de guía para el resto del artículo es ¿Cómo las prácticas de producción pueden influir en las relaciones entre ambos programas?

El resto del estudio está organizado de la siguiente manera: La Sección 2 proporciona las definiciones e hipótesis. Después en la -Sección 3- se presentan las variables de investigación. La metodología se muestra en la Sección 4. En el epígrafe 5, se discuten los resultados. Finalmente, en el apartado 6 se presentan solo, limitaciones y futuras investigaciones.

\section{DEFINICIONES E HIPÓTESIS}

Existe una tendencia general hacia un aumento

3 http://preview.thenewsmarket.com/Previews/PWC/DocumentAs sets/382375.pdf en el uso de la gestión de tecnológica (TM) en las fábricas, debido a la creencia de correlación positiva que permite aumentar la competitividad al mejorar algunas medidas de rendimiento, por ejemplo, la reducción de costos, la mejora de la calidad o de la flexibilidad. Sin embargo, se critican a menudo estas inversiones, por no crear los resultados deseados, es decir, las iniciativas tecnológicas frecuentemente llevan ambos: 1) La implementación ineficaz de las nuevas prácticas de producción y 2) La competitividad deseada no es alcanzada lo suficientemente rápida. Para esto se entiende que es necesario tener en cuenta que la interconexión entre la tecnología y el rendimiento está influenciada por una serie de factores controlables y no controlables, sin embargo, todos ellos son importantes para el resultado final. Por lo tanto, cuando las dimensiones de tanto la tecnología de productos como de la tecnología de procesos son ampliamente aplicadas en una fábrica, se puede decir que la misma está en camino de alto rendimiento por una visión tecnológica más completa.

Así pues, dado que TM se puede definir como la vinculación entre las áreas de la ingeniería, de las ciencias y de la gestión para planear, desarrollar e implementar capacidades tecnológicas, que permitan ajustar y lograr objetivos estratégicos y operativos de las fábricas (White y Bruton, 2010). Por ello, este artículo tipifica que la fábrica tiene que contar con una visión más progresista y dinámica en el marco de la aplicación de las prácticas y herramientas tecnologías en el sector manufacturero, considerando prácticas de producción, más allá de la tecnología “dura” (p.ej. hardware, equipo, etc.). Por lo tanto, se supone una definición abierta que comprende los aspectos tecnológicos que se vinculan con los aspectos humanos y organizativos, de la forma en que las fábricas operan (Heim y Peng, 2010). Así, este estudio se centrará en dos aspectos principales de la gestión tecnológica, como son: producto y procesos (Trentin et al., 2012; Morita et al., 2011).

8 Revista Economía y Administración (E\&A) / V0L. 8 
Por otro lado, partiendo de que la estrategia de producción es la ejecución de un plan a largo plazo, que permita no sólo el uso de los recursos de una organización, sino como estos se vinculan con la estrategia de negocios (Davis et al., 2005), se puede decir que no existe suficiente investigación empírica amplia que trate el efecto de los niveles de implementación de las prácticas de producción de la estrategia de producción -y mucho menos en relación a publicaciones de competitividad por alto rendimiento-, hay señales claras de que la estrategia de producción puede desempeñar un papel fundamental en la evaluación de las nuevas tecnologías, porque un análisis de la tecnología adecuada puede eliminar muchos riesgos, dado que la tecnología de alto rendimiento es un factor clave para mejorar la competitividad global (Ortega et al., 2015; Machuca et al, 2011).

Por lo tanto, ya que muchos autores consideran que las bases para la generación global de ventajas competitivas provienen de decisiones en la producción, que debe ser enmarcado en la estrategia de producción (PS). Además, la tecnología de gestión (TM) es una parte absolutamente esencial de esta gestión. Así pues, para utilizar con eficacia la estrategia de producción, se deben considerar las capacidades de la tecnología (Ortega et al, 2012; Ortega, 2009). Por lo que, la tecnología puede ser factor limitante de la estrategia de dos maneras: 1) La tecnología existente determina la estrategia que una organización puede seguir; y 2) Una fábrica que desea seguir una estrategia diferente puede necesitar expandir/ajustar su base tecnológica. Así, es claro que tanto la tecnología cómo las estrategias pueden influirse mutuamente, lo que sugiere una relación entre ellos. Sin embargo, todavía no está muy claro el contexto en el que dicho enlace ocurre.

Por lo tanto, la primera hipótesis que se plantea en esta investigación empírica es la siguiente:

H1: Los niveles de implementación de una fábrica con prácticas de producción de PS están relacionados positivamente con los niveles de implementación de las prácticas TM.

Además, con el fin de visualizar caminos de la competitividad, el presente trabajo incluye métodos complementarios para probar dos clases de fábricas -competitivas por alto rendimiento y el resto consideradas como de rendimiento estándar-, teniendo en cuenta la competitividad o en la industria electrónica, por lo tanto, se prueba la siguiente hipótesis:

H2: Las fábricas competitivas tienen mayores niveles de implementación de prácticas de producción de PS, así como prácticas de TM, que el resto de las fábricas.

\section{VARIABLES DE INVESTIGACIÓN}

Con el fin de poner en práctica el marco de referencia y las hipótesis en la sección anterior, a continuación, se presentan algunas variables de investigación divididas en tres categorías: 1) Cuatro prácticas para el programa de PS; 2) Cuatro prácticas para el programa $T M$; y 3) Competitividad, medida por medio del rendimiento multidimensional de operaciones.

\subsection{Estrategia de producción (PS)}

Para tratar cómo los recursos de la organización se deben configurar y así lograr los objetivos de negocios, este estudio considera las siguientes cuatro prácticas de producción a largo plazo de la estrategia de producción.

\subsubsection{Interrelación de la estrategia} de producción con la de negocios. Representa la coherencia entre la estrategia de fabricación y la estrategia de negocios. Con dicha coherencia se verifica si la estrategia de negocios se traduce en producción. 


\subsubsection{Planificación estratégica formal.} Esto significa el grado en el que los planes para la toma de decisiones se formalizan, por las acciones tomadas por la dirección. Esto se evidencia por la existencia de una misión escrita, metas a largo plazo y estrategias para sus implementaciones.

\subsubsection{Comunicación de estrategia} de producción. Esta práctica de producción mide los esfuerzos gerenciales para comunicar a lo largo de las fábricas: estrategia, metas y objetivos competitivos.

\subsubsection{Anticipación de nuevas} tecnologías. A medida que las nuevas tecnologías se vuelven disponibles, se cree que las fábricas que anticipan su disponibilidad están en mejores condiciones para implementarlas y sacarle provecho como una fuente de ventaja competitiva. Por lo tanto, esta práctica determina el estatus de adecuación de la fábrica, en el avance de descubrimientos tecnológicos para participar en la implementación de nuevas tecnologías cuando estén disponibles.

\subsection{Gestión tecnológica (TM).}

Las siguientes cuatro prácticas de producción se consideran en este estudio para planear, desarrollar e implementar capacidades tecnológicas de las fábricas.

\subsubsection{Aplicación eficaz del proceso.} Esta práctica representa si la fábrica implementa apropiadamente una nueva tecnología de proceso/ producción, después de haberla adquirida.
3.2.2. Esfuerzos de diseño interfuncionales. Representa el nivel y la cantidad de entradas/insumos que el departamento de producción tiene a bien resolver en el nuevo proceso de introducción del producto. Incluye la cooperación y la coordinación a través de las divisiones funcionales.

\subsubsection{Cooperación en introducción de} nuevos productos. Esta práctica de producción representa el tipo de proceso utilizado en la introducción de productos.

\subsubsection{Participación de proveedores.} Representa si la fábrica trabaja en estrecha colaboración con su cartera de proveedores en el desarrollo de nueva tecnología y optimización de procesos.

3.3. Competitividad: Índices de rendimiento (P)

La última categoría de variables se refiere a los índices de rendimiento competitivo de la fábrica, con relación a los competidores globales en la industria. Ellos fueron evaluados subjetivamente por cada gerente de fábrica, en una escala Likert de cinco puntos. Los siguientes nueve índices de desempeño se incluyen en tres objetivos fundamentales en el departamento de producción, reflejadosdelasiguientemanera: 1) Costos -coste unitario de fabricación; 2) Calidad (conformidad a las especificaciones del producto); y 3) Capacidad de respuesta -tiempo del ciclo, plazo de ejecución de desarrollo, rendimiento de entrega a tiempo, lanzamiento a tiempo de nuevos productos, flexibilidad para cambiar la mezcla de productos, flexibilidad para cambiar el volumen. 


\section{METODOLOGÍA}

\subsection{Datos y medición}

El estudio se basa en el análisis estadístico utilizando la base de datos del proyecto internacional Producción de Alto Rendimiento, por sus siglas en inglés (HPM), que contempla una encuesta que involucró a fábricas de nueve países, ubicados en América, Asia y Europa.

La unidad de análisis son empresas pertenecientes al sector de la manufactura electrónica, con un mínimo de 100 trabajadores como requisito. Por lo tanto, los datos utilizados para el análisis posterior fueron tomados de 88 fábricas parte de este rubro, registradas al azar. A cada una de estas 88 empresas se les aplicó un instrumento compuesto por 12 cuestionarios, dirigidos a los diferentes puestos de la fábrica, que van desde el gerente general hasta operadores. Estos cuestionarios examinaron los temas de producción para las ocho prácticas (cuatro para PS y cuatro para TM) y nueve índices de rendimiento a través de más de 40 diferentes ítems (preguntas).

Todas las preguntas fueron contestadas mediante escalas Likert. Cada práctica se conceptualizó como un factor de primer orden y medida a través de un conjunto de distintos elementos -validez de contenido y del constructo, y fiabilidad fueron probados- siendo significativos mucho más alto que el punto de corte de (Kim y Mueller; Ford et al., 1978; Cronbach, 1951).

Respecto al rendimiento $(\mathrm{P})$, se construyó una medida de capacidad de respuesta que refleja el logro de una fábrica a partir de la velocidad, fiabilidad y dimensiones de entrega (Ortega y Eguia, 2010). Por lo tanto, el costo, la calidad y capacidad de respuesta se utilizaron para observar el total de la eficacia.

Todos los resultados de medición correspondientes estarán disponibles bajo petición.

\subsection{Métodos}

Para responder a las hipótesis de la Sección 2, se utilizará como marco de referencia al ajuste bivariado entre los componentes expuestos con anterioridad -Epígrafe 3-, al analizar cómo las prácticas de producción de TM y PS están relacionadas (Ortega et al., 2011). Se considera al ajuste como la consistencia de dos o más factores, por lo que un buen ajuste entre factores relevantes debería mejorar las condiciones productivas de las fábricas.

El estudio de este ajuste se fundamenta en las prácticas específicas de TM vinculadas a distintas prácticas de PS. No se pretende que la dirección de causalidad deba ser identificada con el modelo utilizado, no obstante, al evaluar la articulación de estos elementos se comprueba si hay algún ajuste entre los niveles de implementación de las prácticas de estrategia de fabricación y las de gestión tecnológica.

Un primer modelo de ajuste que se utilizará para el estudio de datos de la prueba de $\mathrm{H} 1$ es el enfoque de selección (Meilich, 2006), por medio del análisis de correlación canónica -CCA-, dado que es adecuado para el propósito de esta investigación, ya que se basa en la suposición de que los niveles de implementación de un proceso de fabricación deben ser regulados y/o adaptados, teniendo en cuenta el nivel de otro proceso de fabricación y viceversa, para que dicho proceso de fabricación sea controlado/mejorado.

Complementario al anterior, se propone un segundo modelo de ajuste, interacción, para el testeo de H2, con la idea de evaluar los cambios producidos en el rendimiento por los efectos del ajuste (o desajuste). Técnicas tales como Análisis de Sub Grupo (SGA), y análisis de correspondencia múltiple (MCA), son algunos de los esquemas analíticos adecuados. Estas técnicas no tienen por objeto determinar la dirección de la causalidad de las dos variables principales, 
pero sirve para probar el tipo de ajuste entre ellos en diferentes entornos de rendimiento. Esto permite conceptualizar cómo cada tipo/clase de fábrica (Alto Rendimiento/ HP vs. Rendimiento Estándar/SP), como subgrupo, considera el ajuste bivariado de las prácticas en estudio, para la relación que se fijará entre las prácticas de PS y las de TM.

La Tabla 1 resume los dos métodos de análisis conforme las dos hipótesis:

Tabla 1. Método de análisis e hipótesis

\begin{tabular}{llll} 
Paso & Hipótesis & Método & Variables \\
\hline 1 & H1 & CCA & Independientes: 4 prácticas PS y 4 de TM \\
2 & H2 & SGA; MCA & $\begin{array}{l}\text { Independientes:_4 prácticas PS y 4 de TM. } \\
\text { Dependiente: } 3 \text { índices de rendimiento para } \\
\text { subgrupos de dos clases de fábricas (Hp y SP) }\end{array}$ \\
\hline
\end{tabular}

Fuente: Elaboración propia

\section{DISCUSIÓN DE RESULTADOS}

La primera prueba fue el ajuste selección, es decir, si existen implementaciones comunes de todas las prácticas juntas de TM y PS. La Tabla 2 muestra estos resultados, hechos por el análisis de correlación canónica.

Tabla 2. PS y TM: Análisis de correlación canónica

\begin{tabular}{|l|c|}
\hline & Primera variable canónica \\
\hline Correlación canónica & 0.762 \\
\hline $\mathrm{R}^{2}$ & 0.580 \\
\hline Nivel de significancia & 0.000 \\
\hline Índice de redundancia: PS & 0.372 \\
\hline Índice de redundancia: TM & 0.276 \\
\hline Cargas canónicas PS & \\
\hline Planificación estratégica formal & $\mathbf{0 . 7 5 8}$ \\
\hline Anticipación de nuevas tecnologías & $\mathbf{0 . 9 1 9}$ \\
\hline Comunicación de estrategia de producción & $\mathbf{0 . 4 9 2}$ \\
\hline Interrelación estrategia de producción con la de negocios & $\mathbf{0 . 0 8 2}$ \\
\hline Cargas canónicas TM & $\mathbf{0 . 9 6 5}$ \\
\hline Implementación de procesos eficaz & $\mathbf{0 . 5 5 0}$ \\
\hline Esfuerzos de diseño INTERFUNCIONALES & $\mathbf{0 . 6 0 7}$ \\
\hline Nuevo producto cooperación introducción & 0.152 \\
\hline Participación del proveedor & \\
\hline
\end{tabular}

Fuente: Carga mínima de 0.31 para niveles de significancia de 0.05 (Graybill, 1961)

12 Revista Economía y Administración (E\&A) / V0L. 8 
La primera correlación canónica se sitúa en 0.762 -que es alta-. Además, el índice de redundancia muestra que alrededor de un tercio de la variación en los índices PS y TM se explica por la primera canónica entre las variables en análisis. Específicamente, el índice de redundancia en PS es 0,372 , mientras que la tecnología tiene el índice de redundancia de 0.276. Estos valores de redundancia están cercanos, lo que apunta a que las variaciones están siendo compartidas, si se acepta la primera función canónica.

Todas las prácticas de producción del PS están significativamente relacionadas con las variables canónicas de TM -las variables canónicas representan prácticas-, a excepción de participación del proveedor. En el análisis se puede destacar que, en términos generales, esta relación es justificable como desarrollo e implementación de la gestión tecnológica y por lo tanto deberá de ser estratégica para el sector industrial en cuestión contemplada dentro de la estrategia de producción.

En un nivel general, se puede mencionar que las prácticas de PS tienen relaciones con las prácticas de TM, por una implementación común de sus prácticas en la fábrica. Por tanto, es válido inferir sobre la existencia de un efecto bidireccional, el cual podría manifestarse entre las prácticas de ambos programas -PS y TM-, con la salvedad de la práctica de producción de participación del proveedor. Por lo tanto, se acepta la hipótesis $\mathrm{H} 1$ "los niveles de implementación de una fábrica con prácticas de producción de PS están relacionados positivamente con los niveles de implementación de las prácticas TM", con esa única reserva.
Después, al incluir las medidas de rendimiento en el análisis, las fábricas se clasifican como sigue. Fábricas de alto rendimiento (HP): si el costo, la calidad y capacidad de respuesta son mayores que sus respectivas medias, y fábricas de rendimiento estándar (SP): lo contrario. Hay 18 HP de las 81 fábricas, que representa el 22,2\%.

A continuación, se realizó el MCA para verificar si la implementación de las prácticas de TM y las prácticas de PS están vinculadas a la clasificación HP/SP considerada. La práctica se considera de "implementación alta" si su valor es más alto que la media para cada industria individualmente. El gráfico de análisis de correspondencia se muestra en la Figura 1 (con 80 fábricas). Excepto por el rendimiento (representado por HP y SP), cada uno de los casos restantes (prácticas) en la Figura 1.

Se representa por un caso de estos datos. Para cada caso de un "Yes", se introduce en la categoría en la que el caso respectivo pertenece ("implementación alta") y un "No" en caso contrario.

Se puede observar en la Figura 1, que las HP están vinculadas a la alta implementación de las prácticas, mientras que las SP están vinculadas a bajo implementación. Esto parecería indicar que las SP no están muy bien caracterizadas por el bajo nivel de implementación de estas prácticas, mientras que las HP están mejor caracterizadas por su alta implementación, dando apoyo a $\mathrm{H} 2$ : "Las fábricas competitivas tienen mayores niveles de implementación de prácticas de producción de PS, así como prácticas de TM, que el resto de las fábricas". 


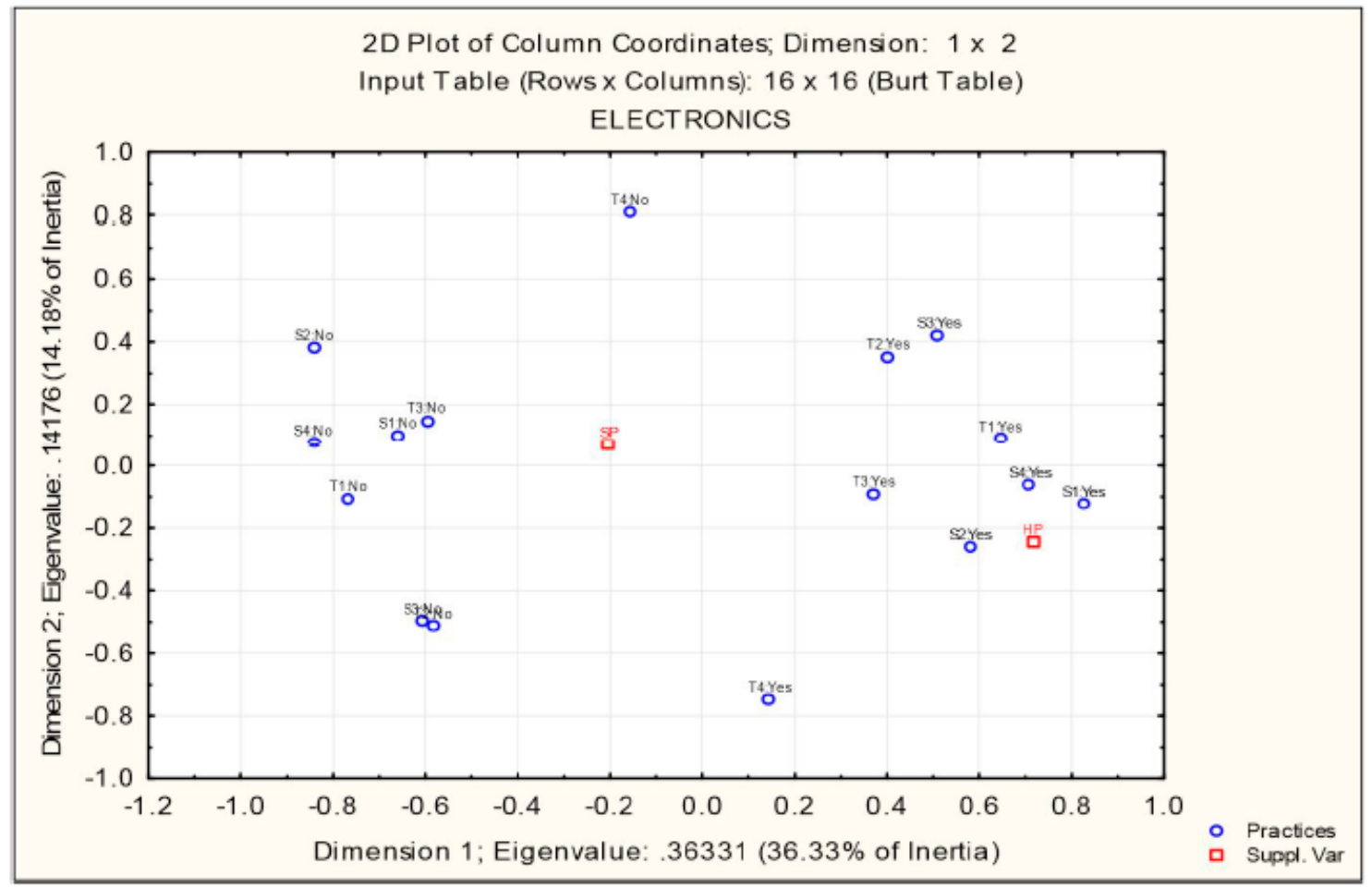

Figura 1. MCA: Gráfico de Tabla Burt

Fuente: (S1: Planificación estratégica formal; S2: Anticipación de nuevas tecnologías; S3: Comunicación de estrategia de producción; S4: Interrelación de la estrategia de producción con la de negocios, T1: Aplicación eficaz del proceso; T2: Esfuerzos de diseño inter-funcionales; T3: Cooperación en introducción de nuevos productos; T4: Participación de proveedores)

\section{CONCLUSIONES}

Los resultados fueron los esperados, ya que muestran la existencia de los vínculos entre las prácticas de producción del programa de estrategia de producción y las prácticas de gestión tecnológica. Sin embargo, usando sólo la supervivencia como medida -ajuste de selección por medio de CCA- es una cantidad que no tiene en cuenta las diferencias entre los datos existentes, ni tampoco asegura que la correlación testeada realmente distingue entre las fábricas existentes -o que han sobrevivido- y las que han fallado (salido del mercado o se han fusionado).

Por lo tanto, el uso de SGA-MCA, como un método complementario, tiene la ventaja de incorporar una medida de resultados, tales como el rendimiento, con el fin de distinguir entre los diferentes niveles de implementación en la relación, de acuerdo con una definición del tipo de fábrica dual -implementación alta o estándar-. Esto demostró que empresas de alto rendimiento tienen ambos clústeres de prácticas implementadas en conjunto, teniendo en cuenta unos con otros.

Estos vínculos podrían deberse a las características específicas del sector electrónico, al tener en cuenta que los diferentes tipos de procesos, prácticas y equipos implican diferentes objetivos, así como estrategias de producción, que pueden impactar en el desarrollo e implementación de la gestión tecnológica de la fábrica.

Como parte de su contribución, este trabajo toma primeros pasos en el testeo para los

14 Revista Economía y Administración (E\&A) / VOL. 8 
ISSN 2219-6722 / ISSNE 2222-2707

Economía y Administración (E\&A), Vol.8 (1) pp.5-16 @ 2017, IIES-UNAH Una Mirada A a Competitividad Global: Producción De La Industria Electrónica CÉSAR H. ORTEGA JIMÉNEZ / PEDRO GARRIDO VEGA / JOSÉ ANTONIO DOMÍNGUEZ MACHUCA

efectos de clase/tipo de fábrica (HP vs SP) entre interrelaciones de prácticas de producción en el ámbito internacional del sector de la electrónica. Esto demuestra, que existen diferencias en los niveles de implementación entre las prácticas propuestas de estrategia de producción y las de gestión tecnológica, que a su vez impactan en el rendimiento y por tanto en la competitividad global de la producción de la industria electrónica.

Debido la limitante de que los datos analizados son exclusivos del sector industrial de electrónica, se presenta oportunidades para una investigación futura sobre los efectos contextuales entre diferentes industrias. Por lo tanto, para una mayor implicación practica y teórica, se puede integrar tanto la teoría de la contingencia de modelos sistémicos y bivariados en las interrelaciones PS-TM, así como los factores contextuales, a la perspectiva del "ajuste" en particular. Finalmente, es importante señalar que es de gran interés, demostrar adicionalmente en investigaciones futuras, el potencial de las prácticas de producción, mediante la incorporación de criterios múltiples de factores debidos a prácticas de infraestructura -como es el caso de este estudio-, sino también de prácticas contextuales, junto con los criterios usado aquí del tipo de fábrica dual -alto rendimiento y el resto-.

\section{RECONOCIMIENTOS}

Esta investigación ha sido financiada parcialmente por el Proyecto Producción de Alto Rendimiento (PAR) del Instituto de Investigaciones Económicas y Sociales de la Universidad Nacional Autónoma de Honduras y, el Ministerio de Ciencia español e Innovación, proyecto DPI2014-60365-R. Los autores desean reconocer a ambas instituciones por su apoyo. Finalmente, una versión anterior en inglés ha sido publicada en el congreso CEAT 2013 


\section{REFERENCIAS}

Aoki, K., Staeblein, T., Tomino, T., 2013. Monozukuri capability to address product variety: A comparison between Japanese and German automotive makers. International Journal of Production Economics. Available online 13 March 2013, ISSN 0925-5273, 10.1016/j. ijpe.2013.02.026.

Cronbach, L.J. 1951. Coefficient alpha and the internal structure of tests. Psychometrika, 16:297-334.

Davis, M. M., Aquilano, N. J., Balakrishnan, J., y Chase, R. B. (2005). Fundamentals of operations management. McGraw-Hill Ryerson, USA.

Ford J.K., Mac Callum R.C., Trait M. 1986. The application of exploratory factor analysis in applied psychology: A critical review and analysis. Personnel Psychology, B(2):291-314.

Garrido P, Ortega Jimenez, C.H. Díez Pérez JL., Morita, M. 2015. Implementation of technology and production strategy practices: Relationship levels in different industries, International Journal of Production Economics, Vol. 161, pp. 201-216.

Graybill, F.A. 1961. An Introduction to Linear Statistical Models, New York: McGraw Hill.

Heim G.R., Peng D.X. 2010. "The impact of information technology use on plant structure, practices, and performance: an exploratory study", Journal of Operations Management, 28(2): 144-162.

Kim J., Mueller C. W. 1978. Introduction to factor analysis. Newbury Park CA: Sage Publications.

Machuca JAD, Ortega-Jiménez C.H., Garrido-Vega P., Pérez Diez de los Ríos J.L. 2011. "Do technology and manufacturing strategy links enhance operational performance? Empirical research in the auto supplier sector", International Journal of Production Economics, 133(2): 541-550.
Meilich O.2006. "Bivariate Models ofFitinContingency Theory. Critique and a Polynomial Regression Alternative", Organisational Research Methods, 9(2): 161-193.

Morita, M., James Flynn, E., Ochiai, S. 2011. Strategic management cycle: The underlying process building aligned linkage among operations practices. International Journal of Production Economics, 133(2): 530-540.

Ortega Jimenez, C. H. O., Machuca, J. A., GarridoVega, P., y Filippini, R., 2015. The pursuit of responsivenessinproductionenvironments:From flexibility to reconfigurability. International Journal of Production Economics, 163, pp. 157172.

Ortega, C. H., Garrido-Vega, P., y Dominguez Machuca, J. A., 2012. Analysis of interaction fit between manufacturing strategy and technology management and its impact on performance. International Journal of Operations \& Production Management, 32(8), pp. 958-981.

Ortega Jiménez, C. H. O., Garrido-Vega, P., \& González, S. G., 2011. Manufacturing strategy-technology relationship among auto suppliers. International Journal of Production Economics, 133(2), pp. 508-517.

Ortega C.H., Eguia I., 2010. "Sistema de Manufactura Reconfigurable y Competitividad Industrial. Economía y Administración (antes Economía Política), 48(2), pp. 97-114.

Ortega C.H., 2009. Vinculo Estrategia de producción Tecnología en la Industria Hondureña: Ajuste de Selección. Economía Política (Ahora Economía y Administración), 47(2): pp. 133-148.

White, M., y Bruton, G. 2010. The management of technology and innovation: A strategic approach ( $2^{\text {a }}$ edición). South-Western College Publishing, USA.

16 Revista Economía y Administración (E\&A) / VoL. 8 